\title{
A Survey of Novel Airborne SAR Signal Processing Techniques and Applications for DLR's F-SAR Sensor
}

\author{
M. Jäger, M. Pinheiro, O. Ponce, A. Reigber, R. Scheiber \\ Microwaves and Radar Institute, German Aerospace Center (DLR) \\ Münchenerstr. 20, 82234 Oberpfaffenhofen, GERMANY \\ e-mail: rolf.scheiber@dlr.de
}

\begin{abstract}
DLR's Microwave and Radar Institute has been operating the F-SAR airborne SAR instrument since 2007. This contribution presents first the SAR imaging capabilities of the system followed by a discussion on recent developments in SAR signal processing using F-SAR data acquired in diverse special modes, each suitable for a specific application: large baseline, dual-frequency SAR interferometry for precise DEM generation, high resolution circular SAR imaging, advanced filtering and polarimetric change detection. The paper concludes with an outlook on future developments.
\end{abstract}

\section{Introduction}

Today satellite SAR images contribute to a variety of operational services in areas of maritime surveillance, security applications and disaster monitoring. Additionally, the different sensors not only support scientific questions related to climate change, like modeling of ice sheets and estimation of forest biomass, but also commercial services for DEM generation or subsidence measurements. Most of these techniques were first demonstrated using airborne SAR data, offering high flexibility for deployment and experiment setup. One of these first airborne SAR sensors in Europe was the E-SAR system of DLR, which was operated from the late 1980's until 2009. Its successor F-SAR is able to acquire fully polarimetric SAR images in five frequency bands - up to three of them simultaneously. FSAR operates in single- and repeat-pass interferometric (incl. Pol-InSAR and tomographic) mode and can acquire and process data along non-rectilinear flight passes (e.g. circular). The most relevant system parameters are summarized in Table 1 and its carrier aircraft is shown in Fig. 1. More details on F-SAR system parameters, the established imaging modes, and essential processing steps are given in [1].

Table 1: F-SAR technical parameters

\begin{tabular}{|c|c|c|c|c|c|}
\hline & $X$ & $\mathrm{C}$ & $\mathrm{S}$ & $\mathrm{L}$ & $\mathrm{P}$ \\
\hline $\begin{array}{l}\text { Centre Frequency } \\
{[\mathrm{GHz}]}\end{array}$ & 9.60 & 5.30 & 3.25 & 1.325 & $\begin{array}{l}0.35 / \\
0.435\end{array}$ \\
\hline $\begin{array}{l}\text { Pulse Bandwidth } \\
\text { [MHz] }\end{array}$ & 760 & 384 & 300 & 150 & 100 \\
\hline $\mathrm{PRF}[\mathrm{kHz}]$ & 5 & 5 & 5 & 10 & 10 \\
\hline $\begin{array}{l}\text { Peak Transmit Power } \\
{[\mathrm{kW}]}\end{array}$ & 2.50 & 2.20 & 2.20 & 0.90 & 0.90 \\
\hline Range Resolution [m] & 0.3 & 0.6 & 0.75 & 1.5 & 2.25 \\
\hline $\begin{array}{l}\text { Azimuth Resolution } \\
{[\mathrm{m}]}\end{array}$ & 0.2 & 0.3 & 0.5 & 0.6 & 1.5 \\
\hline Range Coverage $[\mathrm{km}]$ & & & & 5 & \\
\hline ADC Sampling & \multicolumn{5}{|c|}{$\begin{array}{l}8 \mathrm{Bit} \text { real; } 1 \mathrm{GS} / 500 \mathrm{MS} \text { selectable; } \\
\text { max number of samples } 64 \mathrm{k} / \text { range line; }\end{array}$} \\
\hline Data Rate & \multicolumn{5}{|c|}{$\begin{array}{l}180 \mathrm{MByte} / \mathrm{s} \text { max (per recording } \\
\text { channel); four recording channels }\end{array}$} \\
\hline
\end{tabular}

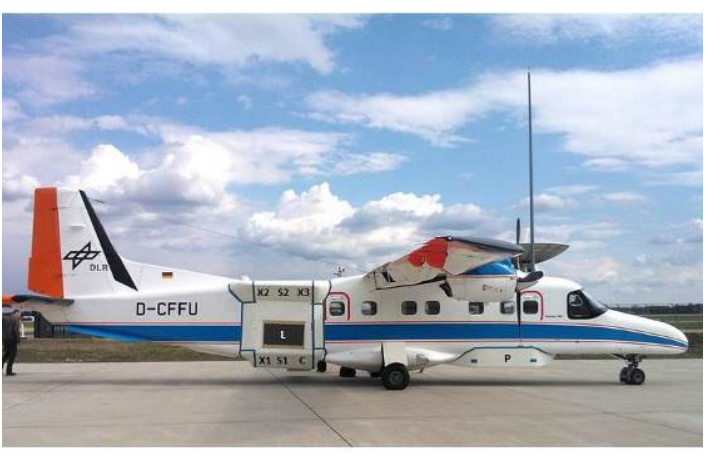

Figure 1. Do-228 aircraft with F-SAR antenna carrier: Each antenna's position is indicated by the frequency band identifier. 
With these characteristics, the system is used to demonstrate innovative applications, which presently cannot be achieved by satellite sensors, first because of the increased spatial resolution and better noise level of the airborne data, and second because of the flexibility inherent to airborne platforms. Each of these applications demands for its specific signal processing algorithms. For supplementing the examples in [1] this paper discusses the following, recently developed application cases:

- Dual-frequency large baseline SAR interferometry for high resolution and high precision digital elevation model (DEM) generation in the German Wadden Sea [2],

- Polarimetric circular SAR imaging for high resolution, multi-angular imaging and enhanced object recognition [3][4],

- High quality speckle filtering for polarimetric data analysis (such as temporal change detection) and interferometric applications [5].

The essential processing steps in the derivation of these data products will be summarized, addressing the challenges encountered in terms of sensor calibration where appropriate. Samples of final F-SAR products are included to illustrate the potential of these novel data processing and information extraction possibilities.

\section{Dual-frequency large baseline SAR interferometry}

Until now, the generation of high resolution Digital Elevation Models (DEMs) from airborne SAR interferometry could not compete with airborne laser scanning (ALS) techniques, primarily due to the small baseline limits imposed by the aircraft dimensions. For comparable height accuracy, in the order of $10 \mathrm{~cm}$, the interferometric baseline must become several tens of meters, which can only be achieved in a repeat-pass scenario. However, in these cases the height of ambiguity (HoA) also reduces to few meters, often resulting in phase unwrapping difficulties. Even if the process can be supported by DEMs derived from single-pass interferometry, phase unwrapping errors still occur because the statistical noise in single-pass DEMs is comparable to the HoA of the repeat-pass pair. Therefore a dual-frequency solution is proposed, which consists in simultaneous acquisition of X-and S-band repeat-pass data. Since the wavelength factor is approximately 3, joint phase unwrapping leads to a stable result, even for isolated areas. Being acquired along the same track, residual motion errors of the data do not impact the robustness of the unwrapping. The processing of dual-frequency data for a high precision DEM follows these steps [2]:

- SAR focusing using topography adaptive motion compensation based on a DEM derived from single-pass interferometry or other coarse resolution reference [1].

- Interferometric processing including residual motion error estimation by means of multi-squint. The residual errors estimated with X-band are applied also to S-band [1].

- Filtering of phases with total variation (TV) filter.

- Joint unwrapping of X- and S-band phases.

- Correction of constant and linear baseline errors based on few reference points. The correction is performed in complex domain to avoid biased estimates due to potential phase unwrapping errors [2].

- Fusion of X- and S-band DEMs: A common low-pass error component for the repeatpass phases is estimated from the single-pass phases, which are assumed free of bias. Afterwards the final height is computed using a maximum a posteriori approach, 

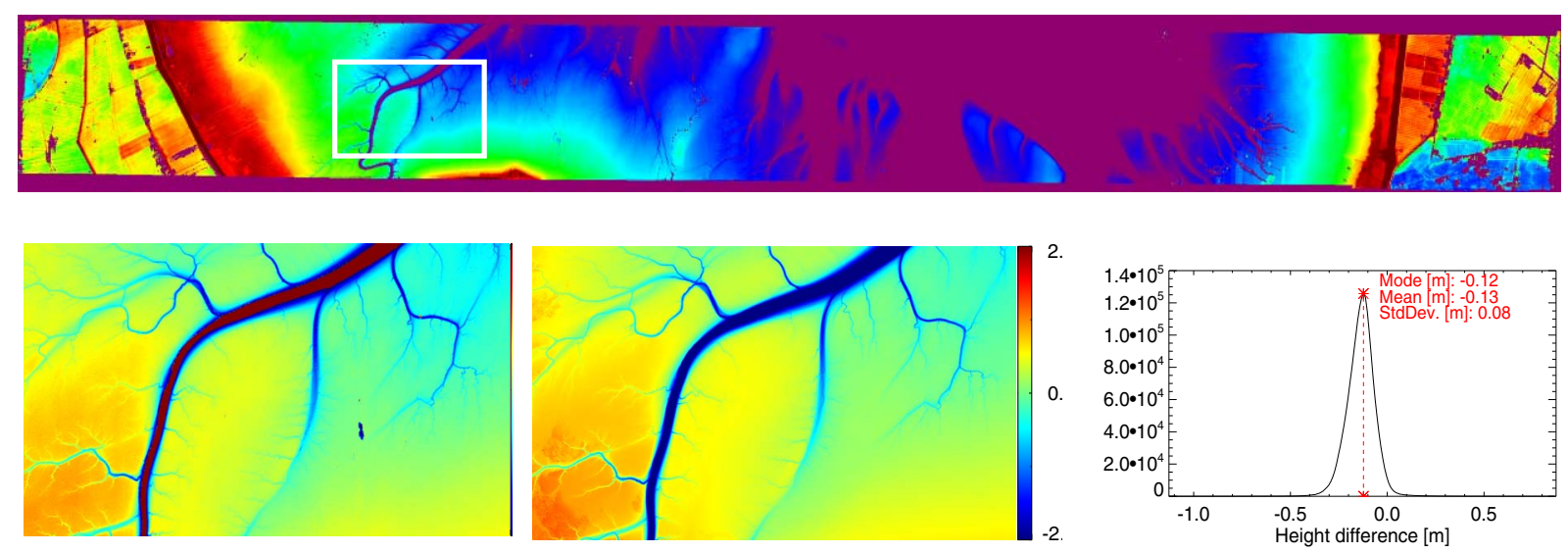

Figure 2. High precision Digital Surface Model of part of Jade Bight at the German North Sea. The complete $20 \mathrm{~km}$ long swath with non-valid / water areas in violet (top) and zoom of dry-fallen area (bottom,left) including comparison with ALS data (middle) and histogram of differences (right).

which constrains the solution to the smallest HoA, i.e. the one of the X-band repeat-pass acquisition.

The methodology has been applied for computing a DEM over the Jade Bight in North Germany. Compared to ALS techniques, a much larger area can be mapped within the short time frame of low tide. F-SAR was acquiring simultaneously X- and S-band data along two parallel tracks at $2400 \mathrm{~m}$ altitude and separated by $20 \mathrm{~m}$ in vertical and horizontal. This ensured HoA in between $1.5 \mathrm{~m}$ to $6 \mathrm{~m}$ in S- and 0.5 to $2 \mathrm{~m}$ in X-band. The fused DEM and a comparison with an ALS DEM acquired 3 months earlier are presented in Fig.2.

\section{Circular SAR Imaging}

Circular SAR offers multiple aspect imaging and very high resolution possibilities for isotropic targets. Fig. 3 presents a color composite derived from a L-band fully polarimetric circular FSAR data set acquired on May 21, 2014 near the Bavarian village of Erlstaett. The geometric accuracy and resolution $(<0.06 \mathrm{~m})$ after focusing are verified on the basis of a Luneburg lens located within the test site (the violet dot in zoom 1). Since man-made structures, such as power pylons, are not present in the DEM underlying the image formation, their ground projection into a surrounding circular area constitute an unambiguous signature that can be used for detection (see zoom 2). Other types of man-made and natural features can be identified more easily compared to the traditional stripmap SAR mode because of the $360^{\circ}$ angular observation (e.g. the cattle in zoom 3 and the power lines in zoom 4, but also buildings and fences).

F-SAR's ability to acquire SAR data along circular tracks has recently been extended to recording of data along multiple circles separated in height [3]. In addition to single image, combining data from multiple circles enables better sidelobe suppression and improved height resolution, allowing multi-angular 3-D imaging. Depending on the final aim, the processing can be performed following different methodologies. The first possibility is to focus the individual SAR images on a geographic grid, using a fast factorized back-projection algorithm adapted to circular SAR geometries (full aperture images) and a high precision DEM. The process is repeated for several height offsets, thus providing 3-D imaging, e.g. of a forest. The second possibility computes subaperture images and combines them in a tomographic mode. Thus, aspect angle dependent 3-D models can be generated (Holographic SAR tomography). For the tomographic processing standard Fourier beamforming, generalized likelihood ratio test (GLRT) and compressed sensing (CS) approaches are used. 

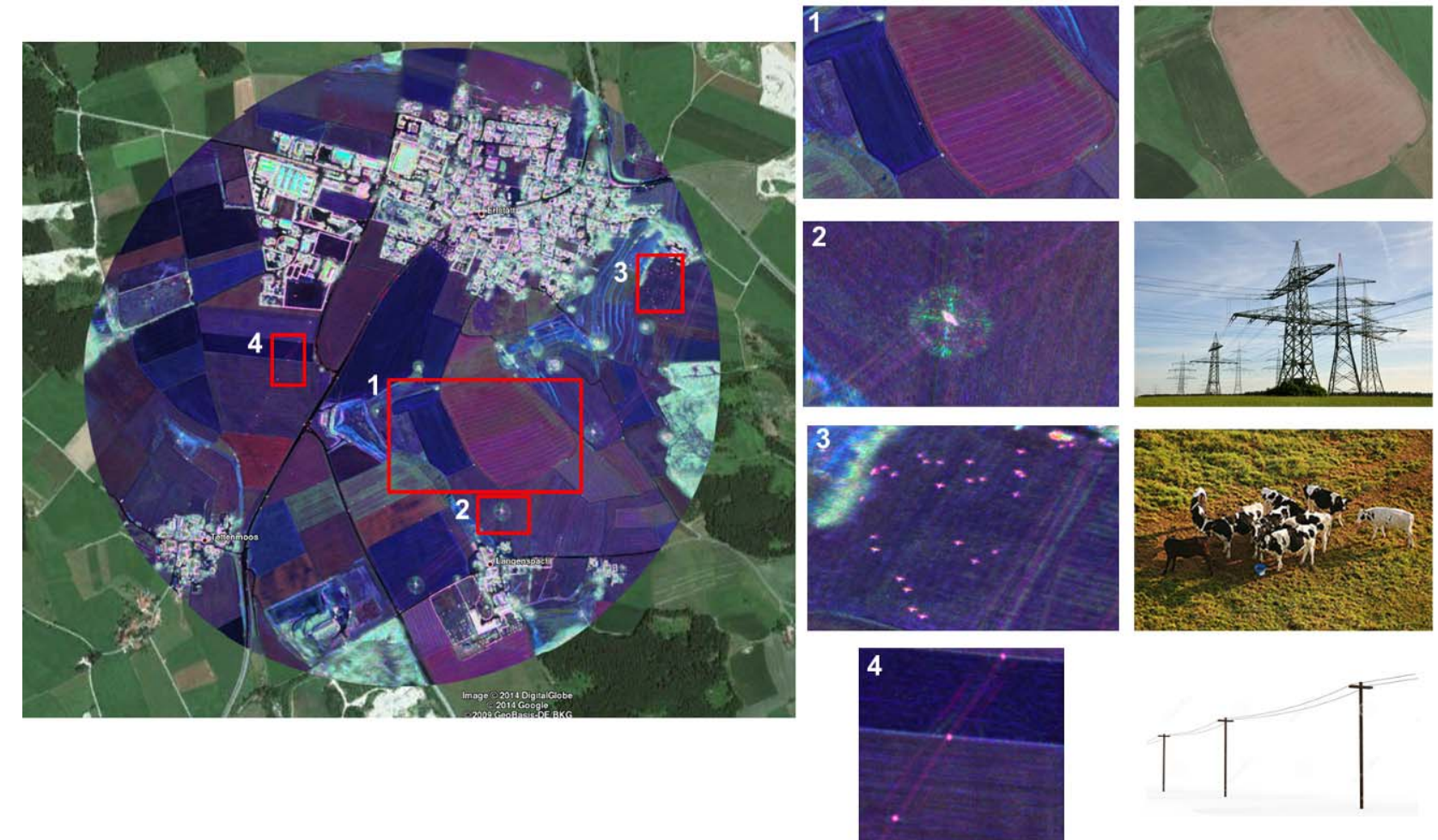

Figure 3. A fully polarimetric L-band circular SAR image in the Pauli basis (image diameter $1.8 \mathrm{~km}$, spatial sampling $6 \mathrm{~cm}$ ). Zooms 1 to 4 depict an agricultural area, high-voltage pylons, a cattle herd and a low voltage power line, respectively.

Comparative results for a forest using these methodologies can be found in [3][4]. Presently, ice scenarios are investigated using recently recorded F-SAR data from Swiss glaciers. Besides that, multiple polarimetric CSAR images can be acquired at different times along nearly identical circles. After convenient processing onto exactly the same grid they can be used for change detection, similar to the case discussed next for F-SAR stripmap data.

\section{Polarimetric Change Detection and Filtering}

F-SAR data support several possibilities for change detection: comparison of (geocoded) SAR intensity images, coherent change detection with interferometric pairs, and polarimetric change detection. In all cases, data pairs or time series with nearly identical imaging geometry is to be preferred. For man-made target change detection, the investigations performed so far indicate that polarimetric change detection is the most robust approach, in terms of falsealarm rate, as it is less affected by the temporal decorrelation than coherent, interferometric techniques. The change detector employed for the results presented in this section is that proposed by Conradsen et al. in [6]. This detector is based on the assumption of Wishart distributed polarimetric covariance/coherence matrices and produces two outputs of interest to users:

- The result of applying a test statistic to the generalized Wishart likelihood ratio, see eqn. (19) in [6]. This is a scalar value, for each resolution cell of the input covariance matrices, between 0.0 and 1.0, which can be understood as the probability that a change has taken place (under the assumption of Gaussian statistics of the backscattered field).

- A binary mask obtained by thresholding the above test statistic to yield the actual change detection result. 
The input parameters to the change detector are the effective number of looks (ENL), which typically needs to be tuned to account for the presence of natural textures, as well as the threshold for change detection. In the experiment reported, the threshold has been uniformly fixed at 0.99 (corresponding to a $99 \%$ probability that a change has taken place). The ENL should generally be chosen somewhat lower than the value suggested by truly homogeneous (in the statistical sense) regions in the data to avoid false positive detections by effectively 'widening' the Wishart distribution to account for natural texture.

To mitigate the impact of speckle noise and improve the false alarm rate, we propose to employ a spatially adaptive speckle filter, in particular the polarimetric non-local means filter [5], to reduce speckle noise while preserving spatial resolution. Instead of applying the filter independently to master and slave covariance matrices, we found that using the polarimetric interferometric (PolInSAR) covariance matrix reduces the number of false alarms near sharp edges in the input images (e.g. building or road edges). This approach involves the following processing steps:

- Concatenation of master and slave scattering vectors after cross-polar symmetrization to obtain a single 6-element vector in each resolution cell.

- The outer product of these vectors yields singular 6 times 6 covariance matrices. A $4 \mathrm{x}$ 4 presumming in range $\mathrm{x}$ azimuth then yields full-rank PolInSAR covariance matrices.

- The Pol-InSAR covariance matrices are speckle filtered using the non-local means filter.
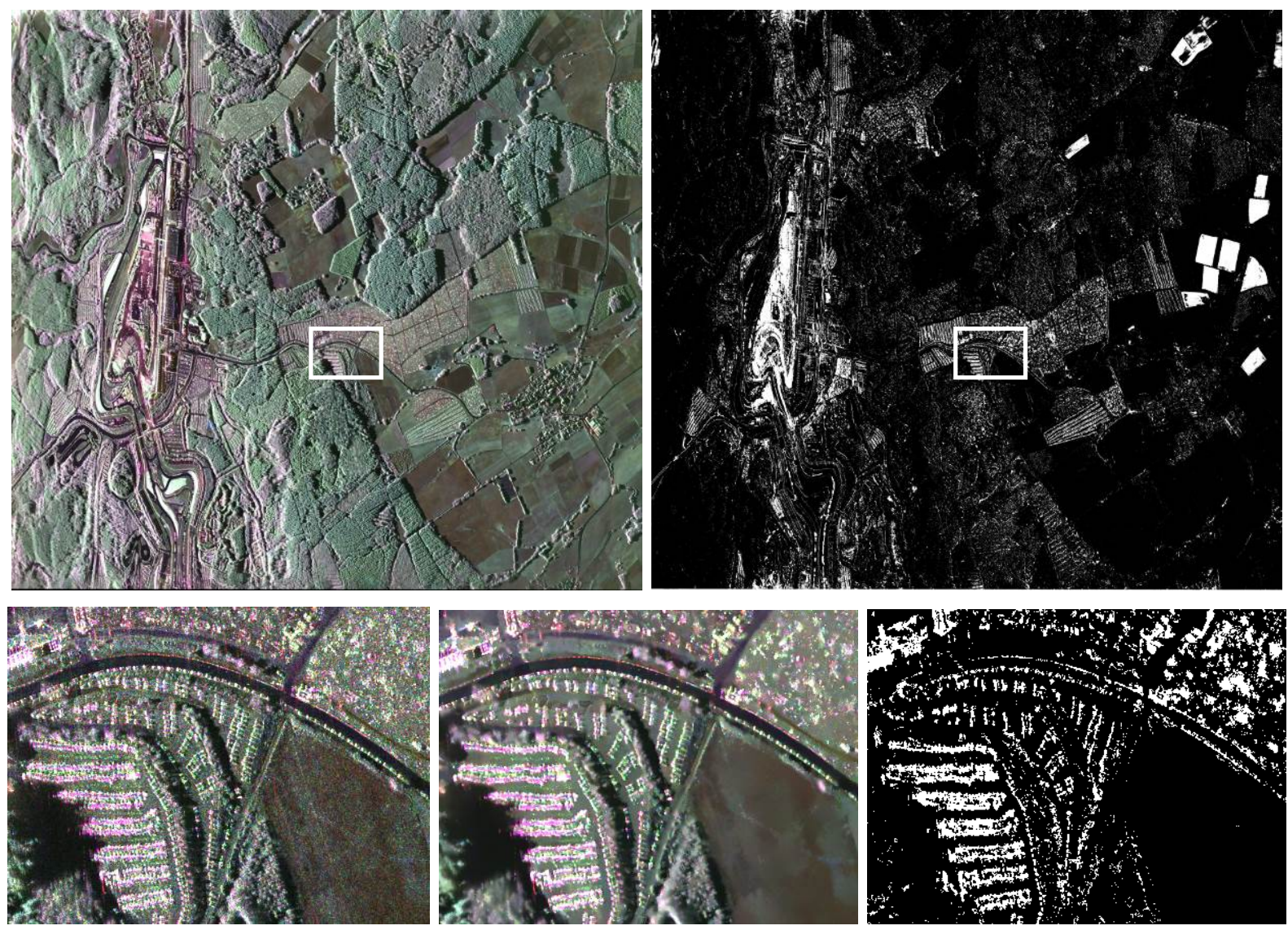

Figure 4. Polarimetric change detection for stepped-frequency X-band data. The F-SAR data were acquired on 2014-05-14 and 2014-06-06 at the Nürburg Ring, before and during the „Rock-am-Ring” festival. Full scene size image of $3 \mathrm{~km}$ by $3 \mathrm{~km}$ and change detection result after thresholding (top). Zoom of white rectrangled area with parking lots (left) and campground (top): original, non-local means filter and change detection result (bottom). 
- The two $3 \times 3$ blocks on the diagonal of the filtered result are then the input data for change detection. For low number of false alarms the ENL is preferably set to 10, which, in this case, is significantly lower than the ENL measured on homogeneous regions of the data.

Fig. 4 exemplifies the performance of the suggested polarimetric change detector using two $\mathrm{X}$-band data sets acquired with a temporal separation of 21 days. Whilst coherence based interferometric change detection will indicate changes over all vegetated areas due to temporal decorrelation, their polarimetric signature usually stays stable, such that the approach outlined above identifies mostly man-made changes. In the example shown, this includes the stage and infrastructure set-ups for the festival, the parking lots and camp grounds, but also some plugged or harvested agricultural fields.

\section{Summary and Future Work}

This paper has summarized some new applications for airborne SAR data, recently demonstrated using the interferometric and polarimetric capabilities of DLR's F-SAR sensor. For each of them new processing strategies and algorithm adaptations were implemented. In the upcoming months extensive F-SAR campaigns are planned in Greenland and West-Africa, which at least in part will provide additional data sets for similar analyses. Future hardware developments include extension to digital beamforming capabilities, which then will allow more technology-oriented demonstrations for future spaceborne SAR missions.

\section{References:}

[1] A. Reigber, R. Scheiber, M. Jäger, P. Prats, I. Hajnsek, Th. Jagdhuber, K. Papathanassiou, M. Nannini, E. Aguilera, St. Baumgartner, R. Horn, A. Nottensteiner and A. Moreira: VeryHigh-Resolution Airborne Synthetic Aperture Radar Imaging: Signal Processing and Applications. Proceedings of the IEEE, 101 (3), pp 759-783, 2013.

[2] M. Pinheiro, A. Reigber, R. Scheiber and M. Jäger: DEM generation using large-baseline airborne InSAR. European Conference on Synthetic Aperture Radar (EUSAR), 2014-06-03 2014-06-05, Berlin, Germany, 2014

[3] O. Ponce, P. Prats, R. Scheiber, A. Reigber, A. Moreira, and E. Aguilera: Polarimetric 3-D Reconstruction from Multi-Circular SAR at P-Band. IEEE Geoscience and Remote Sensing Letters, 11 (4), pp. 803-807, 2014

[4] O. Ponce, P. Prats, R. Scheiber, A. Reigber and A. Moreira: Study of the 3-D Impulse Response Function of Holographic SAR Tomography with Multicircular Acquisitions. European Conference on Synthetic Aperture Radar (EUSAR), 2014-06-02 - 2014-06-05, Berlin, Germany

[5] Ch.Deledalle, L. Denis, F. Tupin, A. Reigber and M. Jäger: NL-SAR: A Unified Nonlocal Framework for Resolution-Preserving (Pol)(In)SAR Denoising. IEEE Transactions on Geoscience and Remote Sensing, 53 (4), pp 2021-2038, 2015

[6] K. Conradsen, A. A. Nielsen, J. Schou, H. Skriver, "A test statistic in the complex Wishart distribution and its application to change detection in polarimetric SAR data," IEEE Transactions on Geoscience and Remote Sensing, , vol.41, no.1, pp.4,19, Jan 2003 